\title{
Duffin-Kemmer-Petiau equation in Riemannian space-times
}

\author{
J. T. Lunardi用, B. M. Pimentelf and R. G. Teixeira传 \\ Instituto de Física Teórica \\ Universidade Estadual Paulista \\ Rua Pamplona 145 \\ 01405-900 - São Paulo, S.P. \\ Brazil
}

\begin{abstract}
It is developed a generalization of the spin 0 representation of Duffin-KemmerPetiau equation to the case of Riemannian space-times using the tetrad formalism.
\end{abstract}

PACS: 11.10.-z, 03.65.Pm

*On leave from Departamento de Matemática e Estatística. Setor de Ciências Exatas e Naturais. Universidade Estadual de Ponta Grossa. Ponta Grossa, PR - Brazil

${ }^{\dagger}$ E-mail: lunardi@ift.unesp.br

${ }^{\ddagger}$ E-mail: pimentel@ift.unesp.br

$\S$ E-mail: randall@ift.unesp.br 


\section{Introduction}

In this work we intend to study the generalization to Riemannian space-times of Duffin-Kemmer-Petiau (DKP) equation in its spin 0 representation. This equation is a convenient relativistic wave equation to describe spin 0 and 1 bosons with the advantage over standard relativistic equations, like Klein-Gordon (KG) and Proca ones, of being linear. As a matter of fact, this equation was developed specifically to fulfill this characteristic, and provide an equation for bosons similar to Dirac spin $1 / 2$ equation.

The first one to propose what is now known as the $16 \times 16 \mathrm{DKP}$ algebra was Petiau [1], a de Broglie's student, who took as starting point the former's work on first order wave equations on $16 \times 16$ matrices that were products of different Dirac matrices spaces. Latter it was showed that this algebra could be decomposed into 5, 10 and 1 degrees irreducible representations, the latter one being trivial [2]. Anyway, Petiau's work remained unkown to the majority of scientific community so that Kemmer, working independently, wrote Proca's equation as a set of coupled first order equations as well as the equivalent spin 0 case [3]. Although these set of equations could be written in $10 \times 10(\operatorname{spin} 1)$ and $5 \times 5($ spin 0$)$ matrix forms, it was not clear which algebraic relations these matrices should obey. Based on this work, Duffin was able to put the equations sets in a first order $\beta$ matrix formulation presenting 3 of the 4 commutation relations present in the DKP algebra [4]. This result provided the motivation to Kemmer to complete formalism and present the complete theory of a relativistic wave equation for spin 0 and 1 bosons [5]. These and other facts related with the historical development of DKP theory, as well as a detailed list of references on the subject, can be found in Krajcik and Nieto paper on historical development of Bhabha first order relativistic equations [6]. 
We will proceed in our work by presenting in section 2 the basic results on DKP equation on Minkowski space-time. We shall not enter in details of DKP theory but simply quote the most important results and properties necessary to the understanding of this work. For further details we suggest the reader to original works [1, 田, 可 or classic textbooks [7, 8]. In the next section we will present some basic results in tetrad formalism that are necessary to perform the passage from Minkowskian to Riemannian space-times, made in section 3. Then, in section 4, we will use the results presented previously to develop the generalization of DKP theory to Riemannian space-times. Finally, we present our conclusions and comments in section 5. Throughout this work we will adopt the signature $(+---)$ to the metric tensors as well as Einstein implicit summation rule, except otherwise stated. Moreover, Latin letters will be used when labelling indexes concerned to the Minkowski space-time or to the Minkowskian manifolds tangent to the Riemannian manifold, while Greek letters will label indexes referred to the Riemannian manifold. Both Latin and Greek indexes will run from 0 to 3, except when we clearly state the opposite.

\section{DKP equation in Minkowski space-time}

The DKP equation is given by

$$
\left(i \beta^{a} \partial_{a}-m\right) \psi=0
$$

where the matrices $\beta^{a}$ obey the algebraic relations

$$
\beta^{a} \beta^{b} \beta^{c}+\beta^{c} \beta^{b} \beta^{a}=\beta^{a} \eta^{b c}+\beta^{c} \eta^{b a}
$$

being $\eta^{a b}$ the metric tensor of Minkowski space-time. This equation is very similar to Dirac's equation but the algebraic properties of $\beta^{a}$ matrices, which have no 
inverses, make it more difficult to deal with. From the algebraic relation above we can obtain (no summation on repeated indexes)

$$
\left(\beta^{a}\right)^{3}=\eta^{a a} \beta^{a},
$$

so that we can define the matrices

$$
\eta^{a}=2\left(\beta^{a}\right)^{2}-\eta^{a a}
$$

that satisfy

$$
\begin{gathered}
\left(\eta^{a}\right)^{2}=1, \eta^{a} \eta^{b}-\eta^{b} \eta^{a}=0 \\
\eta^{a} \beta^{b}+\beta^{b} \eta^{a}=0(a \neq b), \\
\eta^{a a} \beta^{a}=\eta^{a} \beta^{a}=\beta^{a} \eta^{a} .
\end{gathered}
$$

With these results we can write the Lagrangian density for DKP field as

$$
\mathcal{L}=\frac{i}{2} \bar{\psi} \beta^{a} \overleftrightarrow{\partial}_{a} \psi-m \bar{\psi} \psi
$$

where $\bar{\psi}$ is defined as

$$
\bar{\psi}=\psi^{\dagger} \eta^{0}
$$

From this Lagrangian we can obtain DKP equation through a variational principle. Moreover, we will choose $\beta^{0}$ to be hermitian and $\beta^{i}(i=1,2,3)$ anti-hermitian so that the equation for $\bar{\psi}$ can also be easily obtained by applying hermitian conjugation to equation (11).

Besides that, under a Lorentz transformation $x^{\prime a}=\Lambda_{b}^{a} x^{b}$ we have

$$
\begin{gathered}
\psi \rightarrow \psi^{\prime}=U(\Lambda) \psi, \\
U^{-1} \beta^{a} U=\Lambda_{b}^{a} \beta^{b},
\end{gathered}
$$

which gives, for infinitesimal transformations $\Lambda^{a b}=\eta^{a b}+\omega^{a b}\left(\omega^{a b}=-\omega^{b a}\right)$ [7],

$$
U=1+\frac{1}{2} \omega^{a b} S_{a b}, S_{a b}=\left[\beta_{a}, \beta_{b}\right] .
$$


If one uses two sets of Dirac matrices $\gamma^{a}$ and $\gamma^{\prime a}$ acting on different indexes of a 16 component $\psi$ wave function it can be verified that the matrices

$$
\beta^{a}=\frac{1}{2}\left(\gamma^{a} I^{\prime}+I \gamma^{\prime a}\right)
$$

satisfy the algebraic relation (2), but these matrices form a reducible representation since it can be shown [5, [] that this algebra has 3 inequivalent irreducible representations: a trivial 1 degree $\left(\beta^{a}=0\right)$ without physical significance; a 5 degree one, corresponding to a 5 component $\psi$ that describes a spin 0 boson; and a 10 degree one, corresponding to a 10 component $\psi$ describing a spin 1 boson.

The spin 0 sector can be selected from a general representation through the operators

$$
P=-\left(\beta^{0}\right)^{2}\left(\beta^{1}\right)^{2}\left(\beta^{2}\right)^{2}\left(\beta^{3}\right)^{2},
$$

which satisfies $P^{2}=P$, and

$$
P^{a}=P \beta^{a}
$$

It can be shown [7] that

$$
P^{a} \beta^{b}=P \eta^{a b}, P S_{a b}=0
$$

and, as consequence, under infinitesimal Lorentz transformations (12) we have

$$
P U \psi=P \psi
$$

so that $P \psi$ transforms as a (pseudo)scalar. Similarly

$$
P^{a} U \psi=P^{a} \psi+\omega_{b}^{a} P^{b} \psi
$$

showing that $P^{a} \psi$ transforms like a (pseudo)vector.

Applying these operators to DKP equation (1) we have

$$
\partial_{a}\left(P^{a} \psi\right)=\frac{m}{i} P \psi,
$$


and

$$
P^{b} \psi=\frac{i}{m} \partial^{b}(P \psi)
$$

which combined provide

$$
\partial_{a} \partial^{a}(P \psi)+m^{2}(P \psi)=\square(P \psi)+m^{2}(P \psi)=0
$$

These results show that all elements of the column matrix $P \psi$ are scalar fields of mass $m$ obeying KG equation while the elements of $P_{a} \psi$ are $\frac{i}{m}$ times the derivative with respect to $x^{a}$ of the corresponding elements of $P \psi$. Moreover, we can choose a 5 degree irreducible representation of the $\beta^{a}$ matrices in such a way that

$$
P \psi=P\left(\begin{array}{c}
\psi_{0} \\
\psi_{1} \\
\psi_{2} \\
\psi_{3} \\
\psi_{4}
\end{array}\right)=\left(\begin{array}{c}
0_{4 \times 1} \\
\psi_{4}
\end{array}\right), P_{a} \psi=\left(\begin{array}{c}
0_{4 \times 1} \\
\psi_{a}
\end{array}\right)
$$

so that equation (20) results in

$$
\psi_{a}=\frac{i}{m} \partial_{a} \psi_{4}
$$

allowing us to make $\psi_{4}=\sqrt{m} \varphi$ and obtain

$$
\psi=\left(\begin{array}{c}
\frac{i}{\sqrt{m}} \partial_{a} \varphi \\
\varphi
\end{array}\right), \square \varphi+m^{2} \varphi=0
$$

making clear that the DKP equation describes a scalar particle.

In this work we will center our attention on spin 0 fields so we will work specifically with a 5 rowed representation of $\beta^{a}$. 


\section{Passage to Riemannian space-times}

Before constructing the DKP equation in Riemannian $\mathcal{R}^{4}$ space-times we will construct the tensor quantities on $\mathcal{R}^{4}$ using the tensors defined on a Minkowski manifold tangent to each point of $\mathcal{R}^{4}$, and for this purpose we will use the formalism of "tetrads". Here we will just mention the necessary fundamental results and point out the reader to standard textbooks [10].

A tetrad is constituted by a set of four vector fields $e_{\mu}{ }^{a}(x)$ that satisfy, at each point $x$ of $\mathcal{R}^{4}$, the relations

$$
\begin{gathered}
\eta^{a b}=e_{\mu}{ }^{a}(x) e_{\nu}{ }^{b}(x) g^{\mu \nu}(x), \\
g_{\mu \nu}(x)=e_{\mu}{ }^{a}(x) e_{\nu}{ }^{b}(x) \eta_{a b},
\end{gathered}
$$

and

$$
\begin{aligned}
& \eta_{a b}=e^{\mu}{ }_{a}(x) e^{\nu}{ }_{b}(x) g_{\mu \nu}(x), \\
& g^{\mu \nu}(x)=e^{\mu}{ }_{a}(x) e^{\nu}{ }_{b}(x) \eta^{a b},
\end{aligned}
$$

where

$$
e^{\mu}{ }_{a}(x)=g^{\mu \nu}(x) \eta_{a b} e_{\nu}{ }^{b}(x),
$$

the Latin indexes being raised and lowered by the Minkowski metric $\eta^{a b}$ and the Greek ones by the metric $g^{\mu \nu}$ of the manifold $\mathcal{R}^{4}$.

The components $B^{a b}$ in $\mathcal{M}^{4}$ of a tensor $B^{\mu \nu}$ defined on $\mathcal{R}^{4}$ are given by

$$
B^{a b}=e_{\mu}{ }^{a} e_{\nu}{ }^{b} B^{\mu \nu}, B_{a b}=e^{\mu}{ }_{a} e^{\nu}{ }_{b} B_{\mu \nu},
$$

or inversely

$$
B^{\mu \nu}=e^{\mu}{ }_{a} e^{\nu}{ }_{b} B^{a b}, B_{\mu \nu}=e_{\mu}{ }^{a} e_{\nu}{ }^{b} B_{a b},
$$

and it is easy to see that $A_{\mu} B^{\mu}=A_{a} B^{a}$. 
The Lorentz covariant derivative $D_{\mu}$ is defined as

$$
D_{\mu} B^{a}=\partial_{\mu} B^{a}+\omega_{\mu}^{a} b(x) B^{b}
$$

such that $D_{\mu} B^{a}$ transforms like a vector under local Lorentz transformations $x^{a}=\Lambda_{b}^{a}(x) x^{b}$ on the $\mathcal{M}^{4}$ tangent manifold, where the connection $\omega_{\mu}^{a b}(x)$ on $\mathcal{M}^{4}$ satisfies the transformation rule

$$
\omega_{\mu}^{\prime a b}=\Lambda_{c}^{a} \omega_{\mu l}^{c}\left(\Lambda^{-1}\right)^{l b}-\left(\partial_{\mu} \Lambda\right)^{a}{ }_{c}\left(\Lambda^{-1}\right)^{c b} .
$$

Requiring $D_{\mu}\left(B^{a} A_{a}\right)=\partial_{\mu}\left(B^{a} A_{a}\right)$, since $B^{a} A_{a}$ is a scalar, one gets

$$
D_{\mu} B_{a}=\partial_{\mu} B_{a}-\omega_{\mu}^{b}{ }_{a}^{b} B_{b} .
$$

The total covariant derivative $\nabla_{\mu}$ of a quantity $B_{\nu}{ }^{a}$ will then be

$$
\nabla_{\mu} B_{\nu}^{a}=D_{\mu} B_{\nu}^{a}-\Gamma_{\mu \nu}^{\alpha} B_{\alpha}^{a}
$$

or

$$
\nabla_{\mu} B^{\nu a}=D_{\mu} B^{\nu a}+\Gamma_{\mu \alpha}{ }^{\nu} B^{\alpha a}
$$

where $\Gamma_{\mu \nu}{ }^{\alpha}$ is the connection on the $\mathcal{R}^{4}$ manifold. Then, the requirement that $\nabla_{\mu} e_{\nu}^{a}=0$ relates the connections $\omega_{\mu}^{a b}$ and $\Gamma_{\mu \nu}^{\alpha}$ by

$$
\omega_{\mu}^{a b}=e_{\alpha}^{a} e^{\nu b} \Gamma_{\mu \nu}^{\alpha}-e^{\nu b} \partial_{\mu} e_{\nu}^{a} .
$$

Moreover, the metricity condition $\nabla_{\mu} g^{\nu \alpha}=0$ will imply that $\omega_{\mu}^{a b}=-\omega_{\mu}^{b a}$. Besides this, it is easy to see that $\nabla_{\mu} A^{\mu}=\nabla_{a} A^{a}$, where $\nabla_{a}=e^{\mu}{ }_{a} \nabla_{\mu}$.

\subsection{Generalized DKP equation}

As a first step to the generalization of DKP equation we will generalize the matrices $\beta^{a}$ defined on flat Minkowski manifold $\mathcal{M}^{4}$ obeying equation (2) to matrices $\beta^{\mu}$ defined on Riemannian manifold $\mathcal{R}^{4}$ by

$$
\beta^{\mu}=e^{\mu}{ }_{a} \beta^{a}
$$


that will satisfy

$$
\beta^{\mu} \beta^{\nu} \beta^{\alpha}+\beta^{\alpha} \beta^{\nu} \beta^{\mu}=\beta^{\mu} g^{\nu \alpha}+\beta^{\alpha} g^{\nu \mu}
$$

as it can be shown using the properties of $\beta^{a}$ and equation (28).

Moreover, under a local infinitesimal Lorentz transformation on $\mathcal{M}^{4}$, the multicomponent field $\psi$ will transform as given by equations (10) and (12) so that its variation will be

$$
\delta \psi=\frac{1}{2} \omega_{a b} S^{a b} \psi
$$

and the variation of the tetrad vectors will be

$$
\delta e_{\mu}^{a}=\omega_{b}^{a} e_{\mu}^{b}
$$

If we consider two nearby points $x_{1}$ and $x_{2}$ with the local tetrads $e_{\mu}{ }^{a}\left(x_{1}\right)$ and $e_{\mu}{ }^{a}\left(x_{2}\right)$ then $\psi\left(x_{1}\right)$ and $\psi\left(x_{2}\right)$ are the field $\psi$ referred to these tetrads, respectively. Performing a parallel displacement from $x_{1}$ to $x_{2}$ on the tetrad $e_{\mu}^{a}\left(x_{1}\right)$ we get a new one denoted by $e_{\mu}^{\prime}{ }^{a}\left(x_{2}\right)$ and the field $\psi$ at $x_{2}$ with respect to this new tetrad will be $\psi^{\prime}\left(x_{2}\right)$. Then, the covariant differential of the field can be defined as

$$
D \psi=d x^{a} \nabla_{a} \psi=d x^{\mu} \nabla_{\mu} \psi=\psi^{\prime}\left(x_{2}\right)-\psi\left(x_{1}\right)
$$

or

$$
D \psi=\psi\left(x_{2}\right)-\psi\left(x_{1}\right)-\left[\psi\left(x_{2}\right)-\psi^{\prime}\left(x_{2}\right)\right]
$$

where we separated the translation term $\psi\left(x_{2}\right)-\psi\left(x_{1}\right)$ from the local Lorentz transformation term $\psi^{\prime}\left(x_{2}\right)-\psi\left(x_{2}\right)$. Explicitly we have

$$
\psi\left(x_{2}\right)-\psi\left(x_{1}\right)=d x^{a} \partial_{a} \psi=d x^{\mu} \partial_{\mu} \psi
$$

and

$$
\psi\left(x_{2}\right)-\psi^{\prime}\left(x_{2}\right)=-\frac{1}{2} \omega_{a b} S^{a b}
$$


so that

$$
D \psi=d x^{a} \partial_{a} \psi+\frac{1}{2} \omega_{a b} S^{a b} \psi
$$

From the expression (34) for the Lorentz covariant derivative we see that the variation of the tetrad vector $e_{\mu}{ }^{a}$ under Lorentz transformations on the $\mathcal{M}^{4}$ manifold is

$$
\delta e_{\mu}^{a}=\omega_{\nu}{ }^{a}{ }_{b} e_{\mu}^{b} d x^{\nu}
$$

so, comparing with equation (41) we can identify

$$
\omega^{a b}=\omega_{\nu}^{a b} d x^{\nu}
$$

and rewrite (46) as

$$
D \psi=d x^{\mu}\left(\partial_{\mu}+\frac{1}{2} \omega_{\mu a b} S^{a b}\right) \psi
$$

and the covariant total derivative of field $\psi$ will be

$$
\nabla_{\mu} \psi=\left(\partial_{\mu}+\frac{1}{2} \omega_{\mu a b} S^{a b}\right) \psi
$$

Applying the hermitian conjugation to this expression and using the definition of $\bar{\psi}$ we get

$$
\nabla_{\mu} \bar{\psi}=\partial_{\mu} \bar{\psi}-\frac{1}{2} \omega_{\mu a b} \bar{\psi} S^{a b}
$$

Now we can write the generalized expression for the $\psi$ field Lagrangian

$$
\mathcal{L}=\sqrt{-g}\left[\frac{i}{2}\left(\bar{\psi} \beta^{\mu} \nabla_{\mu} \psi-\nabla_{\mu} \bar{\psi} \beta^{\mu} \psi\right)-m \bar{\psi} \psi\right]
$$

that can be written explicitly as

$$
\begin{aligned}
\mathcal{L}= & \sqrt{-g}\left[\frac { i } { 2 } \left(\bar{\psi} \beta^{\mu}\left(\partial_{\mu} \psi+\frac{1}{2} \omega_{\mu a b} S^{a b} \psi\right)\right.\right. \\
& \left.\left.-\left(\partial_{\mu} \bar{\psi}-\frac{1}{2} \omega_{\mu a b} \bar{\psi} S^{a b}\right) \beta^{\mu} \psi\right)-m \bar{\psi} \psi\right]
\end{aligned}
$$


So we have

$$
\frac{\partial \mathcal{L}}{\partial \bar{\psi}}=\sqrt{-g}\left[\frac{i}{2}\left(\beta^{\mu} \partial_{\mu} \psi+\omega_{\mu a b} \beta^{\mu} S^{a b} \psi-\omega_{\mu}{ }^{\mu}{ }_{b} \beta^{b} \psi\right)-m \psi\right],
$$

and

$$
\begin{gathered}
\partial_{\nu} \frac{\partial \mathcal{L}}{\partial\left(\partial_{\nu} \bar{\psi}\right)}=-\frac{i}{2}\left[\partial_{\nu}\left(\sqrt{-g} \beta^{\nu}\right) \psi+\sqrt{-g} \beta^{\nu} \partial_{\nu} \psi\right], \\
\partial_{\nu} \frac{\partial \mathcal{L}}{\partial\left(\partial_{\nu} \bar{\psi}\right)}=-\frac{i}{2}\left[\sqrt{-g} \omega_{\mu}^{\mu}{ }_{a} \beta^{a} \psi+\sqrt{-g} \beta^{\nu} \partial_{\nu} \psi\right],
\end{gathered}
$$

where we used the result [10]

$$
\partial_{\nu}\left(\sqrt{-g} e_{a}^{\nu}\right)=\sqrt{-g} \omega_{b}^{b}{ }_{a}=\sqrt{-g} \omega_{\mu}^{\mu}{ }_{a}
$$

valid for a Riemannian manifold.

Combining these results we get the generalized DKP equation of motion

$$
i \beta^{\mu} \nabla_{\mu} \psi-m \psi=0
$$

Now we can define the generalized projectors $P^{\mu}$ as

$$
P^{\mu}=e^{\mu}{ }_{a} P^{a}=e^{\mu}{ }_{a} P \beta^{a}=P \beta^{\mu},
$$

where $P$ is given by equation (14) in terms of the matrices $\beta^{a}$. Using equations (16) and (28) it is easy to verify that

$$
P^{\mu} \beta^{\nu}=P g^{\mu \nu}, P S^{\mu \nu}=0
$$

It can be verified also that $P \nabla_{\mu} \psi=\nabla_{\mu}(P \psi)$ and $P^{\nu} \nabla_{\mu} \psi=\nabla_{\mu}\left(P^{\nu} \psi\right)$. Applying these operators to the generalized DKP equation (58) we get

$$
P^{\mu} \psi=\frac{i}{m} \nabla^{\mu}(P \psi)=\frac{i}{m} \partial^{\mu}(P \psi),
$$

and

$$
\nabla_{\mu}\left(P^{\mu} \psi\right)=\frac{m}{i}(P \psi)
$$


As $P \psi$ is a scalar under Lorentz transformation on the Minkowski manifold $\mathcal{M}^{4}$ it will also be a scalar under general coordinate transformations on the Riemannian manifold $\mathcal{R}^{4}$. Similarly, $P^{a} \psi$ is a vector under Lorentz transformations so that $P^{\mu} \psi$ will also be a vector under general coordinate transformations [10]. Combining equations (61) and (62) we get the generalized KG equation

$$
\nabla_{\mu} \nabla^{\mu}(P \psi)+m^{2}(P \psi)=\nabla_{a} \nabla^{a}(P \psi)+m^{2}(P \psi)=0
$$

Using the specific choice of the matrices $\beta^{a}$ that satisfies condition (22) we get as result

$$
\begin{gathered}
P \psi=\left(\begin{array}{c}
0_{4 \times 1} \\
\psi_{4}
\end{array}\right), P^{\mu} \psi=e^{\mu a} P_{a} \psi=e^{\mu a}\left(\begin{array}{c}
0_{4 \times 1} \\
\psi_{a}
\end{array}\right) \\
\psi=\left(\begin{array}{c}
\frac{i}{\sqrt{m}} \nabla_{a} \varphi \\
\sqrt{m} \varphi
\end{array}\right), \nabla_{\mu} \nabla^{\mu} \varphi+m^{2} \varphi=0
\end{gathered}
$$

These results make clear that the generalized DKP equation (58) describes a scalar particle on a Riemannian manifold. Finally, we note that it is possible to make a change in the representation of $\beta$ matrices in such a way that the form of DKP field $\psi$ becomes

$$
\psi \rightarrow \psi_{R}=\left(\begin{array}{c}
\frac{i}{\sqrt{m}} \nabla_{\mu} \varphi \\
\sqrt{m} \varphi
\end{array}\right)
$$

\section{Conclusions and comments}

In this work we presented the generalization of the spin 0 representation of DKP theory by constructing the Riemmanian quantities from the flat space-time ones through the use of tetrad formalism. We have obtained a first order relativistic wave equation that describes a spin 0 particle coupled to the gravitational field. We also showed the complete equivalence between this approach and the usual 
result obtained through KG equation for spin 0 bosons in Riemannian spacetimes. It should be also mentioned that the form (65) for DKP field when the matrices $\beta$ satisfy condition (64) is equivalent to the result obtained when we consider the interaction with eletromagnetic field: the "gradient" components of free field case (the first four components) are replaced by the covariant derivatives [9].

The perspectives for future developments are diverse and some of them are currently under our study. We could mention the extension of this approach to the spin 1 representation of DKP theory and its application to the eletromagnetic field in Riemannian space-times. Moreover, it is interesting to look for the construction of a generalization similar to the one proposed by Dirac to its electron equation [11]. Subsequently, it comes the generalizations to Riemann-Cartan space times and to teleparallel descripition of gravity [12] as a step to compare the question of coupling of DKP fields to torsion in both theories, similarly to what has been done to usual spin 0 and 1 formalisms [13, 14. Independently, it will be analysed the quantic processes in DKP theory with gravitation viewed as an external field, as it has already been done with DKP field in an external eletromagnetic field [15].

\section{Acknowlegdments}

J.T.L. and B.M.P. would like to thank CAPES's PICDT program and CNPq, respectively, for partial support. R.G.T. thanks CAPES for full support. The authors also wish to thank Professor V. Ya. Fainberg by his critical reading of our manuscript. 


\section{References}

[1] G. Petiau, University of Paris thesis (1936). Published in Acad. Roy. de Belg., Classe Sci., Mem in $8^{\circ} 16$ (1936), No. 2.

[2] J. Géhéniau, Acad. Roy. de Belg., Classe Sci., Mem in $8^{\circ} 18$ (1938), No. 1.

[3] N. Kemmer, Proc. Roy. Soc. A166 (1938), 127.

[4] R. J. Duffin, Phys. Rev. 54 (1938), 1114.

[5] N. Kemmer, Proc. Roy. Soc. A173 (1939), 91.

[6] R. A. Krajcik and M. M. Nieto, Am. J. Phys. 45 (1977), 818.

[7] H. Umezawa, "Quantum Field Theory," North-Holland, 1956.

[8] A. I. Akhiezer and V. B. Berestetskii, "Quantum Electrodynamics," Interscience, 1965.

[9] J. T. Lunardi, B. M.Pimentel, R. G. Teixeira and J. S. Valverde - In preparation.

[10] V. de Sabbata and M. Gasperini, "Introduction to Gravitation," World Scientific, 1985.

[11] P. A. M. Dirac, "Max Planck Festschrift," page 339, Veb Deutscher Verlag der Wissenschafter, 1958.

[12] J. W. Maluf, J. Math. Phys. 35 (1994), 335.

[13] V. C. de Andrade and J. G. Pereira, Gen. Rel. Grav. 30 (1998), 263.

[14] V. C. de Andrade and J. G. Pereira, Int. J. Mod. Phys. D8 (1999), 141.

[15] V. Ya. Fainberg, D. M. Gitman and B. M. Pimentel - In preparation. 